\title{
O abandono do tratamento da tuberculose e a atuação do profissional enfermeiro: um olhar sobre as referências
} The abandonment of tuberculosis treatment and professional nursing actions: a look at the
references

El abandono del tratamiento de la tuberculosis y la actuación del profesional enfermero: un vistazo a las referencias

Douglas Rodrigues da Silva ${ }^{1}$; Magdalena Souza da Silva ${ }^{2}$ Tarciso Feijó da Silva ${ }^{3}$; Vitor Ventura Tomé ${ }^{4}$; Lilian Moreira do Prado ${ }^{5}$; Thiago Francisco Neves ${ }^{6}$.

\section{Resumo}

Os objetivos deste artigo foram revisar na literatura os fatores que contribuem para abandono do tratamento da tuberculose e caracterizar as ações da equipe multiprofissional da Atenção Primária, com enfoque para o profissional enfermeiro para diminuição do abandono ao tratamento.A revisão de literatura foi utilizada como metodologia para produção deste artigo de natureza descritiva e os dados foram analisados considerando duas categorias previamente elaboradas. Como resultados foi possível observar que diferentes fatores contribuem para o abandono do tratamento da tuberculose, dentre eles destacamos os aspectos sócio-demográficos, o uso de drogas, o alcoolismo, a associação com outras doenças crônicas, em especial o HIV e o acesso dos usuários aos serviços de saúde. Percebeu-se, ainda, de acordo com as referências analisadas fragilidade na formação dos enfermeiros, sobrecarga deste profissional e trabalho em equipe incipiente. Concluímos que a visita domiciliar, a humanização, o acolhimento e a educação em saúde foram identificados como ferramentas para diminuição do abandono da tuberculose.

Descritores: Enfermagem. Tuberculose. Atenção Primária à Saúde. Recusa ao tratamento.

\footnotetext{
Abstract

The objectives of this article was to review the literature on the factors that contribute to abandonment of tuberculosis treatment and characterize the actions of the multidisciplinary team of primary care, focusing on the professional nurse to decrease abandonment to treatment. The literature review was used to produce this descriptive and the data were analyzed considering two

${ }^{1}$ Graduado em Enfermagem pela Universidade Estácio de Sá. Enfermeiro na Clínica da Família Augusto Boal/SMSRJ. Avenida Guilherme Maxwell, 901. Bonsucesso, Rio de Janeiro, RJ, Brasil, 21040-212. Email: drdouglasrodrigues@yahoo.com.br.

${ }^{2}$ Graduada em Enfermagem pela Universidade Estácio de Sá. Enfermeira no CMS Flávio do Couto Vieira/SMSRJ. Rua Lúcio José Filho s/n. Parque Anchieta, Rio de Janeiro, RJ, Brasil, 21635-290. Email: magdalenasouza.silva@gmail.com ${ }^{3}$ Mestre em Saúde Pública pela Escola Nacional de Saúde Pública Sérgio Arouca. Docente na Universidade Estácio de Sá e Enfermeiro na Policlínica Piquet Carneiro/UERJ. Av. Marechal Rondon, 381. São Francisco Xavier, Rio de Janeiro, RJ, Brasil, 20950-003. Email: tarcisofeijo@yahoo.com.br.

${ }^{4}$ Graduado em Enfermagem pela Universidade Estácio de Sá. Enfermeiro no CMS Nagib Jorge Farah/SMSRJ. Praça Michael Cheib, s/n. Jardim América, RJ, Brasil, 21240-780. Email: vitor.tom@ hotmail.com.

${ }^{5}$ Mestre em Enfermagem pela Universidade Federal do Estado do Rio de Janeiro. Docente na Universidade Estácio de Sá e enfermeira no Instituto Nacional de Cardiologia/Ministério da Saúde. Rua das laranjeiras, 374. Laranjeiras, Rio de Janeiro, RJ, Brasil, 22240-006. Email: pradolilian@ gmail.com.

${ }^{6}$ Graduando em enfermagem pela Universidade Estácio de Sá. Campus Norte Shopping. Av. Dom Hélder Câmara, 5474. Cachambi, Rio de Janeiro, RJ, Brasil, 20771-004. Email: thiagonevesjw@ gmail.com.
} 
ISSN 2179-6750

previously developed categories. It could be observed that there are different factors that contribute to the abandonment of tuberculosis treatment, highlighting the socio-demographic aspect, drug use, alcoholism, association with other chronic diseases, especially HIV and user access to health services. It was noticed also, according to the references analyzed, weakness in nurse's training, overload in nurses and incipient teamwork. Home visits, humanization, greeting and health education emerge as tools for reduction of tuberculosis abandonment.

Key-words: Nursing. Tuberculosis. Primary Health Care. Refusal to treatment.

\section{Resumen}

Los objetivos de este artículo fue revisar la literatura sobre los factores que contribuyen al abandono del tratamiento de la tuberculosis y caracterizar las acciones del equipo multidisciplinario de la atención primaria, centrándose en el profesional de enfermería para disminuir el abandono del tratamiento.La revisión de la literatura se utilizó como metodología para la producción de este enfoque descriptivo y los datos se analizaron teniendo en cuenta dos categorías desarrolladas anteriormente. Como resultado se observó que diferentes factores contribuyen al abandono del tratamiento de la tuberculosis entre los Estos incluyen sociodemográfico, el consumo de drogas, el alcoholismo, la asociación con otras enfermedades crónicas, especialmente el VIH y el acceso de los usuarios a los servicios de salud. Se observó también, de acuerdo con las referencias debilidad analizados en la formación de enfermeras, esta sobrecarga joven equipo profesional y laboral. Llegamos a la conclusión de que la visita a la casa, la humanización, acogedor y educación para la salud se identificaron como herramientas para la reducción del abandono de la tuberculosis.

Palabras-claves: Enfermería. La tuberculosis. Atención primaria de salud. La negativa al tratamiento.

\section{Introdução}

A Tuberculose (TB) é transmitida pelo ar e pode atingir todos os órgãos do corpo. A pessoa infectada pelo Mycobacterium tuberculosis - agente etiológico - libera gotículas no ar iniciando o processo de transmissão da doença. O pulmão é o principal órgão do corpo acometido pela doença, considerando o tropismo do agente etiológico pelas áreas do corpo com muito oxigênio ${ }^{1}$. Os indivíduos que vivem em condições de extrema pobreza, que possuem baixa escolaridade, os alcoólatras, em situação prisional, com coinfecção tuberculose/HIV são os mais suscetíveis à doença ${ }^{2}$. A TB constitui um problema global de saúde pública devido à alta prevalência em muitos países, dentre eles o Brasil, e tem estreita ligação com as condições socioeconômicas ${ }^{3}$, e estas por sua vez, impedem o efetivo controle da doença ${ }^{4}$. Estudo recente realizado no Município de Ribeirão Preto (SP) identificou quanto ao resultado do tratamento cura com variação de $62,7 \%$ a $76,7 \%$, média de abandono de $4,3 \%$ e óbito por tuberculose de $3,9 \%$.

O diagnóstico da tuberculose está associado à clínica e realização da baciloscopia e o tratamento é realizado através do Tratamento Diretamente Observado (TDO) ${ }^{6}$. Devido à resistência 
ISSN 2179-6750

desenvolvida por determinados pacientes o esquema medicamentoso está em constante modificação e têm sido incentivadas novas pesquisas farmacológicas. A Rifampicina, Isoniazida, Pirazinamida, Etambutol, Estreptomicina e a Etionamida estão entre os fármacos utilizados para tratamento da tuberculose pulmonar ${ }^{7}$.

A partir da década de 40, observou-se uma diminuição no índice de mortalidade de TB no Brasil com o advento do tratamento medicamentoso, entretanto, esse índice não se manteve, devido ao surgimento do $\mathrm{HIV}^{8}$. Políticas públicas específicas nos anos seguintes foram sendo criadas pelo Ministério da Saúde com o intuito de aumentar a cobertura de doenças existentes no território nacional, dentre elas a tuberculose. A partir de 2003 as diferentes esferas de gestão pactuaram o fortalecimento da estratégia de TDO visando cumprimento das metas internacionais existentes (70\% detecção de casos bacilíferos e $85 \%$ de cura) o que contribuiu para priorização da doença como um problema de saúde pública ${ }^{9}$.

A publicação do PNCT (Programa Nacional de Controle da Tuberculose) em 2004 abriu espaço para integração entre os diferentes equipamentos de saúde o que contribui para a efetiva ampliação do acesso ao diagnóstico e ao tratamento ${ }^{10}$. Os maiores desafios do programa relacionam-se à estratégia de TDO, a cobertura das ações de abandono do tratamento e a incrementação das taxas de cura da doença. O abandono do tratamento é compreendido como o não comparecimento do indivíduo em acompanhamento à unidade de saúde por mais de 30 dias consecutivos, após a data aprazada para retorno ${ }^{11}$. No contexto que envolve o abandono do tratamento é importante o estabelecimento de uma rede descentralizada de diagnóstico e tratamento integrada à Atenção Primária à Saúde (APS), visando horizontalizar as atividades de vigilância, prevenção e controle da doença, pela incorporação das mesmas às atribuições das equipes da Estratégia Saúde da Família (ESF), considerada, na perspectiva da APS, a porta de entrada do sistema de saúde ${ }^{12}$.

O abandono do tratamento envolve uma série de fatores e não pode ser atribuída culpabilidade apenas ao doente; devem ser consideradas também questões relacionadas aos serviços de saúde, como a desorganização do trabalho em equipe, demora do atendimento, desumanização, falta de vínculo entre doente e profissional de saúde, ausência de busca ativa ao doente e faltoso, entre outros $^{5}$.Desta forma, considerando a epidemiologia associada à doença e a compreensão da mesma como problema de saúde pública, pensamos ser fundamental produzir reflexão que contribua para repensar a prática das equipes de saúde da família e especificamente do profissional enfermeiro.

Assim, definimos como objetivos deste artigo revisar na literatura os fatores que contribuem para abandono do tratamento da tuberculose e caracterizar as ações da equipe multiprofissional da 237 
ISSN 2179-6750

Atenção Primária, com enfoque para o profissional enfermeiro para diminuição do abandono ao tratamento.

\section{Metodologia}

A revisão crítica da literatura foi utilizada para produção deste artigo de natureza descritiva $^{13}$. Na base de dados da Biblioteca Virtual em Saúde (BVS) realizou-se cruzamento em trio dos seguintes descritores Enfermagem, Tuberculose e Atenção Primária à Saúde. Emergiram da pesquisa 71 publicações. Destas, 17 foram excluídas (06 artigos duplicados e 11 artigos de outro idioma, tendo em vista que optou-se por trabalhar somente com publicações em português). Procedeu-se a leitura exploratória dos títulos e resumos das 54 publicações restantes; e considerando a temática e os objetivos do estudo foram selecionados 13 artigos com acesso livre, texto completo, país de afiliação (Brasil) e publicados após a implantação da PNCT em 2004. Destes, 12 pertenciam à base LILACS e 01 à base SCIELO.

Os artigos remanescentes da busca foram lidos na íntegra e organizados por ano, revista, metodologia, tipo de estudo, local e resultados. Utilizou-se a leitura interpretativa para estabelecer relações, confrontar idéias, refutar ou confirmar opiniões ${ }^{14}$ e o referencial teórico foi utilizado para apoiar a análise de dados que considerou duas categorias previamente selecionadas, a saber: fatores que favorecem o abandono do tratamento de tuberculose e atuação da equipe multiprofissional no Programa de Controle da Tuberculose com ênfase no profissional enfermeiro.

\section{Resultados e Discussão}

A análise das publicações a priori permitiu identificar a formação acadêmica dos autores (Gráfico 01) e a metodologia utilizada pelos mesmos nas diferentes produções (Gráfico 02). Destacamos que entre os autores 38 eram enfermeiros, 04 acadêmicos de enfermagem e apenas dois autores possuíam outra formação profissional (Engenheiro de produção química e médico). 


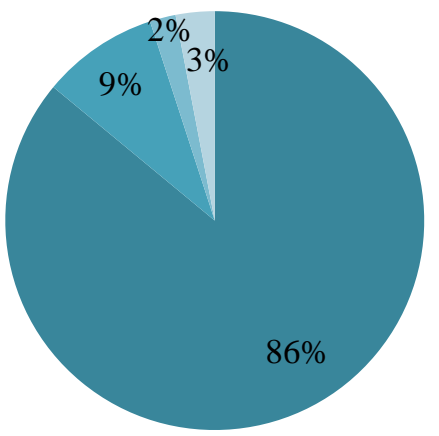

Enfermeiros

- Graduandos de Enfermagem

Engenheiros de Produção Química

Médicos

Fonte: Pesquisa direta

Gráfico 02. Metodologia utilizada pelos autores

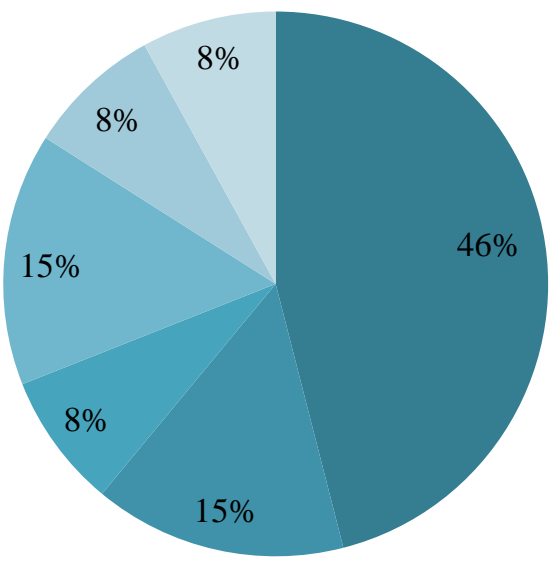

Fonte: Pesquisa direta
Estudos de abordagem qualitativa

- Estudos de abordagem quantitativa e transversal

- Estudo quantitativo de inquérito epidemiológico prospectivo

Estudos quantitativo epidemiológico descritivo

Estudo qualitativo, exploratório, descritivo e representacional

Revisão integrativa

Fatores que favorecem o abandono ao tratamento de tuberculose

Um dos aspectos de maior desafio na luta contra a tuberculose é o abandono do tratamento, pois repercute diretamente na elevação dos índices de mortalidade, incidência da doença e multidrogarresistência ${ }^{15,16}$. Existem vários fatores associados ao abandono do tratamento, dentre eles aspectos sociodemográficos, uso de drogas, relacionados aos serviços de saúde e ao tratamento da doença, ocorrência de outras doenças, principalmente crônicas, e o cuidado em saúde ${ }^{17}$.

A migração dos sintomáticos respiratórios para a atenção secundária e terciária pela dificuldade de acesso à Unidade Básica de Saúde, o que acarreta em um sofrimento físico e emocional tanto para o sintomático respiratório como para os familiares, é apontada como fator que 
ISSN 2179-6750

favorece a prevalência da doença. A dificuldade de acesso atrelada a rigidez de horário das unidades básicas de saúde e limitação no atendimento, as formas de marcação das consultas que em sua grande maioria acontecem de forma presencial, as filas de atendimento nas rotinas dos serviços de saúde e não cumprimento dos horários de atendimento pelos profissionais foram apontados como fatores que contribuem para dificuldade do acesso ${ }^{18}$.A associação com outras doenças crônicas, especificamente a infecção pelo HIV é vista como principal causa para o abandono do tratamento da $\mathrm{TB}^{17}$.

A ausência de capacitação e de instrumentos para atendimento aparecem como fatores que contribuem para acompanhamento inadequado dos casos, já que os profissionais de saúde na prática acabam não seguindo os protocolos preconizados pelas políticas públicas que conversam sobre a doença $^{17,18}$. Dentre os fatores relacionados à disponibilidade e acessibilidade são apontadas como as que mais comprometem o tratamento. Estas são compreendidas não somente como a obtenção da medicação ou de exames diagnósticos protocolados mais de todos os meios, mecanismos e tecnologias que facilitam ou promovem o alcance dos doentes a esses serviços e para que eles permaneçam neles, sem ônus ou custos ao paciente, família e comunidade ${ }^{18}$. Clementino e Miranda (2015) caracterizam a acessibilidade como geográfica, organizacional e financeira e em seu estudo concluíram que estas apresentam-se como barreiras para o tratamento de usuários com diagnóstico de tuberculose pulmonar e extrapulmonar ${ }^{18}$. No entanto,é importante lembrar que o resultado do estudo realizado pelos autores permite identificar fragilidades no PNCT, já que este propõe integração entre os diferentes equipamentos de saúde para efetiva ampliação do acesso ao diagnóstico e ao tratamento ${ }^{10}$.

Por último, a falta de organização das equipes no desenvolvimento das ações de saúde na linha de cuidado da tuberculose é apontada como elemento que corrobora para fragilidade da estratégia de Tratamento Diretamente Observado e abandono do tratamento ${ }^{18}$.

Atuação da equipe multiprofissional no Programa de Controle da Tuberculose com ênfase no profissional enfermeiro

Existem fragilidades no diagnóstico precoce e no cuidado ao doente de TB na Atenção Básica, tal como a deficiência na qualificação profissional, que impede tanto a identificação precoce dos casos quanto as reais necessidades de saúde dos usuários acometidos ${ }^{19}$. Nesse sentido, as instituições formadoras devem auxiliar na formação de profissionais de saúde com habilidades de observar o usuário como sujeito singular, com vivências e histórias a serem consideradas, que avance na compreensão da subjetividade e singularidade, envolvidas no processo de cuidar. Fatores 
ISSN 2179-6750

como a falta de conhecimento sobre a Tuberculose, modo de transmissão e dificuldade nas ações de busca por sintomáticos respiratórios tornam ineficiente o cuidado aos doentes e seus contatos, o que potencializa o risco de transmissão e contribui para o diagnóstico tardio, conduzindo-os para os serviços especializados e para o ambiente hospitalar ${ }^{20}$.

O enfermeiro, realizando ações e atividades gerenciais e assistenciais sem o envolvimento de outra categoria profissional, acarreta em uma atuação restrita, principalmente em relação ao TDO, ao registro de informações no livro verde e à realização de consultas, o que favorece a diminuição dos casos de cura, aumento do abandono, a resistência ao medicamento e, principalmente, a perda da adesão provocada pelo rompimento do vínculo ${ }^{21}$. Este pressuposto é reafirmado devido à debilidade quantiqualitativa do capital humano na Atenção Primária, a sobrecarga de funções e a inadequada qualificação dos profissionais de saúde para lidarem com a $\mathrm{TB}^{22}$. Percebe-se na prática o enfermeiro muito envolvido nas intervenções de controle da tuberculose, geralmente é ele quem direciona as ações, além de assumir para si atribuições que nem sempre são de sua competência. Isso ocorre porque muitos profissionais da enfermagem desconhecem aquilo que realmente é de sua alçada, causando uma sobrecarga laboral em detrimento a um atendimento de qualidade ao paciente ${ }^{20,23,24}$.

O Sistema Único de Saúde (SUS) traz, em suas diretrizes, que a organização dos serviços deve ser pautada pelo princípio da integralidade, onde as ações e serviços devem ocorrer de maneira conjunta e articulada com vistas à prevenção e cura, direcionadas para o indivíduo e para sociedade em todos os níveis de complexidade da rede, com a finalidade de assegurar infra-estruturas contínuas e globais pelos diferentes profissionais, apresentando certa flexibilidade no tempo e espaço, de acordo com a disponibilidade tecnológica local ${ }^{25}$. Daí é importante que cada membro da equipe conheça suas reais atribuições dentro do programa de controle da tuberculose, com a finalidade de fornecer um cuidado integral e, assim, aumentar cada vez mais a probabilidade de resolutividade de suas ações, bem como a criação de vínculos com as pessoas da comunidade, que poderão facilitar a adesão ao tratamento ${ }^{20,26}$.

Embora existam atribuições específicas do profissional enfermeiro na atenção à saúde de usuários existem incontáveis bloqueios assistenciais, tais como acúmulo de funções, inadequada qualificação e visão centralizada e fragmentada da organização das ações de controle da TB, tornando suas ações cada vez mais deficientes no que tange às reais necessidades do usuário portador da $\mathrm{TB}$, fazendo com que haja um crescimento tanto nas taxas de incidências quanto nas de abandono do tratamento ${ }^{20}$. Desta forma, a necessidade de educação permanente com a finalidade de qualificar enfermeiros nos diferentes âmbitos relacionado ao diagnóstico, tratamento e controle da 
ISSN 2179-6750

TB, favorecendo a descentralização das ações de atenção à TB e a implantação da estratégia TDO é vista como essencial $^{20,24}$.

Assim, algumas estratégias apresentam-se como oportunas e devem ser utilizadas pelos enfermeiros para alcançar e identificar primariamente os contatos de tuberculose, como por exemplo: investigar os acompanhantes do paciente no momento da consulta do atendimento diário para o TDO, o incentivo e a supervisão da visita domiciliar realizada pelo Agente Comunitário de Saúde, visando buscar os contatos e orientá-los sobre a importância da realização dos exames; o estímulo a criação de vínculo e de co-responsabilização a partir do TDO por todos os profissionais da unidade de saúde; e a flexibilização no processo de atendimento dos usuários ${ }^{26}$.

A visita domiciliar deve ser valorizada pelo profissional enfermeiro e contribui para uma percepção da realidade de vida da população, permitindo conhecer o ambiente no qual esse indivíduo está inserido. Ao realizar a visita domiciliar por meio da escuta solidária, o portador de TB passa a confiar na equipe e no seu tratamento, o que minimiza os casos de abandono do tratamento e portadores multirresistentes. Além da criação de vínculo com o portador e sua família, a visita domiciliar gera sentimento de segurança, gratidão e reconhecimento da assistência oferecida pelos profissionais da Equipe de Saúde da Família ${ }^{18}$.

O enfermeiro deve envolver a família no tratamento dos usuários com tuberculose ${ }^{23}$. Isto é possível através de práticas educativas, preventivas, curativas, de diálogo, respeito e escuta ${ }^{24}$. A atuação do enfermeiro no controle de tuberculose prevê, ainda, ações de planejamento, organização e avaliação, além da prática do cuidado já inserida neste debate ${ }^{19,20,25}$.

Entender, a tuberculose, enquanto problema de saúde pública traz a tona repensar as práticas em saúde e a realidade do abandono ao tratamento como um grande desafio. Neste estudo foi possível observar que determinados fatores podem contribuir para diminuição da adesão ao tratamento, gerando nos usuários multirresistência às drogas e aumento da transmissibilidade da doença. A análise das referências apontou fragilidades no profissional enfermeiro vinculadas à formação, como déficit de conhecimento sobre a doença e os fatores que influenciam na adesão ao tratamento. A formação acadêmica em questão relaciona-se principalmente à capacidade deste profissional em estabelecer relação na prática dos serviços de saúde no atendimento dos casos de tuberculose com o conhecimento científico, considerando a singularidade de cada indivíduo, os protocolos existentes e o trabalho em equipe. A organização e integração dos serviços de saúde tendo como pressuposto a integralidade da atenção dos usuários apresenta-se como alternativa para lidar com os fatores que contribuem com o abandono do tratamento da tuberculose. 


\section{Considerações Finais}

A humanização e o acolhimento na ESF - considerada como porta preferencial do usuário com tuberculose - apresentam-se como potentes ferramentas para geração de vínculo, inclusão, avaliação de vulnerabilidades e diminuição da equidade. A utilização destas pelos profissionais enfermeiros abre espaço para conhecimento das reais necessidades dos usuários, permite envolvimento dos demais profissionais da ESF, compartilhamento de responsabilidades e contribui para corresponsabilização dos usuários. Outra importante estratégia que pode ser utilizada pelo enfermeiro é a educação em saúde, já que esta permite compreensão sobre a doença, contribuindo para aumento da adesão e diminuição do abandono, além de agregar elementos de prevenção que colaboram a longo prazo para diminuição da incidência e prevalência da doença.

Ademais, reiteramos a necessidade de implicação dos profissionais com as diferentes atividades de prevenção e promoção em saúde para diminuição da transmissão da doença; e da gestão na identificação e análise das fragilidades locais, na programação e no desenvolvimento de atividades, como discussão de caso, que envolvam a realidade de cada equipe de saúde da família, pactuação de melhorias que contribuam para diminuição do abandono da tuberculose e avaliação e monitoramento das ações realizadas.

\section{Referências}

1. Smith DV, Sharma V, Sacchettini JC. TB drug discovery: addressing issues of persistence and resistance.Tuberculosis (Edinb). 2004; 84 (2): 45-55.

2. Rosemberg J. Tuberculose: aspectos históricos, realidades, seu romantismo e transculturação. Bol Pneum Sanit. 1999; 7 (2): 05-29.

3. Mendes AM, Fensterseifer LM. Tuberculose: porque os pacientes abandonam o tratamento? Bol Pneum Sanit. 2004; 12 (1): 25-36.

4. Avelar MCQ, Paula TAC, Shimizu MI, Neves MA, Petrizzo CE. O conhecimento da equipe de enfermagem sobre cuidados com pacientes suspeitos ou portadores de tuberculose pulmonar: estudo exploratório. Online Braz J Nurs [online]. 2006; 5 (2): 01-08.

5. Hino P, Cunha TN, Villa TCS, Santos CB. Perfil dos casos novos de tuberculose notificados em Ribeirão Preto (SP) no período de 2000 a 2006. Ciênc. Saúde Coletiva. 2011; 16 (Supl 1): $1295-1301$

6. Brasil. Ministério da Saúde. Secretaria de Atenção à Saúde. Departamento de Atenção Básica. Vigilância em Saúde: Dengue, Esquistossomose, Hanseníase, Malária, Tracoma e 
ISSN 2179-6750

Tuberculose / Ministério da Saúde, Secretaria de Atenção a Saúde, Departamento de Atenção Básica. - $2^{\mathrm{a}}$ Ed. rev. - Brasília: Ministério da Saúde, 2008.

7. Brasil. Ministério da Saúde. Secretaria de Políticas Públicas. Departamento de Atenção Básica. Coordenação Nacional de Pneumologia Sanitária. Plano estratégico para implementação do plano de controle da tuberculose no Brasil de 2001-2005: Resumo executivo. Brasília: Ministério da Saúde, 2000.

8. Campos R, Pianta C. Tuberculose: histórico, epidemiologia e imunologia, de 1990 a 1999, e co-infecção TB/HIV, de 1998 a 1999, Rio Grande do Sul - Brasil. Bol. da Saúde. 2001; 15 (1): 61-71.

9. Brasil. Ministério da Saúde. Manual de recomendações para o controle de tuberculose no Brasil. Brasília: Ministério da Saúde, 2011.

10. Brasil. Ministério Da Saúde. Secretaria de Vigilância Epidemiológica. Coordenação Geral de Doenças Endêmicas. Programa Nacional de Controle da Tuberculose. Brasília: Ministério da Saúde, 2004.

11. Brasil. Ministério da Saúde. Secretaria de Atenção a Saúde. Departamento de Atenção Básica. Política Nacional de Atenção Básica. Brasília: Ministério da Saúde, 2012.

12. Souza KMJS, PalhaLD, Nogueira PF, Almeida J, Villa TCS, Figueiredo DA. Abandono do tratamento de tuberculose e relações de vínculo com a equipe de saúde da família. Rev Esc Enferm USP. 2010; 44 (4): 904-911.

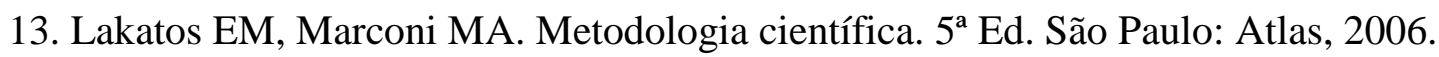

14. Andrade MM. Introdução à metodologia do trabalho científico. $2^{\mathrm{a}}$ Ed. São Paulo: Atlas, 1997.

15. Heringer A, Ferreira VA, Acioli S, Barros ALS. Práticas educativas desenvolvidas por enfermeiros do Programa Saúde da Família no Rio de Janeiro. Rev Gaúcha Enferm. 2007; 28 (4): 01-16.

16. Santos AS, Miranda SMRC. A enfermagem na gestão em atenção primária à saúde. São Paulo: Manole, 2007.

17. Chirinos NEC, Meirelles BHS. Fatores Associados Ao Abandono Do Tratamento Da Tuberculose: Uma Revisão Integrativa. Texto Contexto Enferm. 2011; 20 (3): 599-606.

18. Arcêncio RA, Arakawa T, Oliveira MFDE, Gonzales RIC, Scatena LM, Netto AR, Villa TCS. Barreiras econômicas na acessibilidade ao tratamento da tuberculose em Ribeirão Preto - São Paulo. Rev Esc EnfermUSP. 2011; 45 (5): 1121-1127. 
ISSN 2179-6750

19. Clementino FS, Miranda FAN. Tuberculose: Acolhimento e informação na perspectiva na visita domiciliária. Rev enferm UERJ. 2015; 23 (3): 350-354.

20. Barrêto AJR, Evangelista ALF, Sá LD, Almeida AS, Nogueira JA, Lopes AMC. Gestão do cuidado à tuberculose: da formação à prática do enfermeiro. Rev Bras Enferm. 2013; 66 (6): 847-853.

21. Sobrinho RAS, Souza AL,Wysocki AD, Silva LMC, Beraldo AA, Villa TCS. Conhecimento de enfermeiros de unidades de atenção básica acerca da tuberculose. Cogitare Enferm. 2014;19 (1): 34-40.

22. Rêgo CCD, Macêdo SM, Andrade CRB, Maia VF, Pinto JTJM, Pinto ESG. Processo de trabalho da enfermeira junto à pessoa com tuberculose na atenção primária à saúde. Rev Baiana de Enferm. 2015; 29 (3): 218-228.

23. Oliveira MF, Arcêncio RA, Netto AR, Scatena LM, Palha PF, Villa TCS. A porta de entrada para o diagnóstico da tuberculose no Sistema de Saúde de Ribeirão Preto/SP. Rev Esc Enferm USP. 2011; 45 (4): 898-904.

24. Cecilio HPM, Higarashi IH, Marcon SS. Opinião dos profissionais de saúde sobre os serviços de controle da tuberculose. Acta Paul Enferm. 2015; 28 (1): 19-25.

25. Nogueira JA, Oliveira LCS, Sá LD, Silva CA, Silva DM, Villa TCS. Vínculo e acesso na estratégia saúde da família: percepção de usuários com tuberculose. Rev Rene. 2012; 13 (4): 784-793.

26. Assis EG, Beraldo AA, Monroe AA, Scatena LM, Gonzales RIC, Palha PF, Protti ST, Villa TCS. A coordenação da assistência no controle da tuberculose. Rev Esc Enferm USP. 2012; 46 (1): 111-118. 